\title{
Synthesis of Allenamides by Copper-Catalyzed Coupling of Allenyl Halides with Amides, Carbamates, and Ureas
}

\author{
Barry M. Trost and Dylan T. Stiles \\ Department of Chemistry, Stanford University, Stanford, California 94305-5080 \\ bmtrost@stanford.edu
}

\section{Supporting Information}

\section{Part A: Experimental}

\section{General Considerations:}

Flash chromatography was preformed on silica gel (EM Science, Kieselgel 60, 230-400 mesh, ASTM) using compressed air. TLC was preformed using glass-backed plates coated with $0.2 \mathrm{~mm}$ silica (Merck, DC-Platten, Kieselgel; $60 \mathrm{~F}_{254}$ ). NMR spectra were carried out on a Varian Gemini 200 (200 MHz), Mercury 400 (400 MHz), or Unity $500(500 \mathrm{MHz})$ instruments and are calibrated to residual solvent peaks: proton $\left(\mathrm{CDCl}_{3}\right.$ $7.26 \mathrm{ppm}$ ) and carbon $\left(\mathrm{CDCl}_{3} 77 \mathrm{ppm}\right)$. Optical rotation was measured on a Jasco DIP1000 digital polarimeter in $5 \mathrm{~cm}$ cells at room temperature. All compounds are $>95 \%$ pure by proton NMR unless otherwise noted. All reagents were used as purchased unless otherwise noted. Toluene was dried by passage over a column of alumina. All reactions involving allenes were shielded from ambient light with aluminum foil. Column chromatography on all allenamides was performed with the addition of 5\% triethylamine to the eluting phase.

\section{Experimental Procedures:}

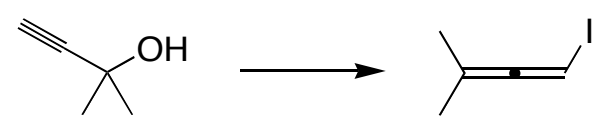

1-iodo-3-methylbuta-1,2-diene was prepared by a modification of the procedure of

Landor et. al. ${ }^{1}$ The original procedure, as followed, gave a product contaminated with a significant amount of 1,3-diene impurities, and in one case, exothermic decomposition upon distillation of the iodide. The following modification avoids distillation.

A $100 \mathrm{~mL}$ roundbottom flask was charged with ammonium iodide (9.93 g, $68.5 \mathrm{mmol}$ ), copper(I) iodide (13.04 g, $68.5 \mathrm{mmol}$ ), copper metal (340 mg, $5.34 \mathrm{mmol}$ ), and 47\% 
hydroiodic acid ( $24.9 \mathrm{~mL}, 37.3 \mathrm{~g}, 137 \mathrm{mmol})$. To this mixture was added, dropwise via an addition funnel, 2-methy-3-butyn-2-ol (6.64 mL, $5.76 \mathrm{~g}, 68.5 \mathrm{mmol})$ in $15 \mathrm{~mL}$ of petroleum ether over the course of $0.5 \mathrm{~h}$. After stirring for $3 \mathrm{~h}$, the biphasic mixture was filtered through glass wool into a separatory funnel. The layers were separated and the aqueous layer was extracted with petroleum ether $(2 \times 15 \mathrm{~mL})$. The organic extracts were combined, washed with water $(2 \times 15 \mathrm{~mL})$, dried over magnesium sulfate, filtered through a plug of silica gel, and concentrated by evaporation to give the allenyl iodide $(5.27 \mathrm{~g}, 39 \%)$ as previously reported. ${ }^{1}$

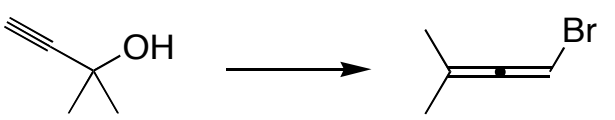

1-bromo-3-methylbuta-1,2-diene was prepared by a similar modification of the procedure of Landor et. al. ${ }^{2}$

A $50 \mathrm{~mL}$ roundbottom flask was charged with ammonium bromide $(2.49 \mathrm{~g}, 25.4 \mathrm{mmol})$, copper(I) bromide ( $3.11 \mathrm{~g}, 22 \mathrm{mmol}$ ), copper metal (158 mg, $2.5 \mathrm{mmol}$ ), and 48\% hydrobromic acid ( $12 \mathrm{~mL}, 17.74 \mathrm{~g}, 105.4 \mathrm{mmol})$. To this mixture was added, dropwise via an addition funnel, 2-methy-3-butyn-2-ol (6.0 mL, $5.208 \mathrm{~g}, 62 \mathrm{mmol})$ in $15 \mathrm{~mL}$ of petroleum ether over the course of $0.5 \mathrm{~h}$. The reaction was heated to $30^{\circ} \mathrm{C}$ for $3 \mathrm{~h}$. The biphasic mixture was filtered through glass wool into a separatory funnel. The layers were separated and the aqueous layer was extracted with petroleum ether $(2 \times 15 \mathrm{~mL})$. The organic extracts were combined, washed with concentrated hydrobromic acid (4 x 8 $\mathrm{mL})$, water $(2 \times 10 \mathrm{~mL})$, dried over magnesium sulfate, filtered through a plug of silica gel, and concentrated by evaporation to give the allenyl bromide $(5.127 \mathrm{~g}, 56 \%)$ as previously reported. $^{2}$

General procedure for synthesis of allenamides:<smiles>CC(C)=CCN1CCOC1=O</smiles> 
An oven-dried $10 \mathrm{~mL}$ roundbottom flask was charged with 2-oxazolidinone (30 $\mathrm{mg}$, $0.345 \mathrm{mmol}), \mathrm{CuTC}$ (4.6 mg, $24 \mu \mathrm{mol})$, and $\mathrm{K}_{3} \mathrm{PO}_{4}(146 \mathrm{mg}, 0.689 \mathrm{mmol})$. After flushing with nitrogen 3 times, $1.5 \mathrm{~mL}$ of toluene was added, followed by $\operatorname{trans}-N, N$ 'dimethylcyclohexyldiamine $(8.1 \mu \mathrm{L}, 7.4 \mathrm{mg}, 52 \mu \mathrm{mol})$, then 1-iodo-3-methylbuta-1,2diene $(70 \mu \mathrm{L}, 114 \mathrm{mg}, 0.587 \mathrm{mmol})$. The flask was covered with aluminum foil and heated to $85^{\circ} \mathrm{C}$ for $7 \mathrm{~h}$. The reaction was cooled and added to $10 \mathrm{~mL}$ of water, then extracted with ethyl acetate $(3 \times 5 \mathrm{~mL})$, washed with brine, dried over magnesium sulfate, and concentrated under vacuum. The residue was purified by column chromatography on silica gel (eluted with 95\% ether, 5\% triethylamine) to afford the allenamide (51 $\mathrm{mg}$, $97 \%$ ) as an oil. $\mathrm{R}_{f}=0.53$ (ether). IR(neat): $2984,2914,1965,1764,1486,1433,1393$, $1310,1251,1082,1035,757,666 \mathrm{~cm}^{-1} .{ }^{1} \mathrm{H} \mathrm{NMR}\left(200 \mathrm{MHz}, \mathrm{CDCl}_{3}\right): \delta 6.58(\mathrm{sp}, J=2.38$ $\mathrm{Hz}, 1 \mathrm{H}), 4.36(\mathrm{dd}, J=9.52,7.87 \mathrm{~Hz}, 2 \mathrm{H}), 3.53(\mathrm{dd}, J=9.34,7.69 \mathrm{~Hz}, 2 \mathrm{H}), 1.78$ (d, $J=$ $2.56 \mathrm{~Hz}) .{ }^{13} \mathrm{C} \mathrm{NMR}\left(50 \mathrm{MHz}, \mathrm{CDCl}_{3}\right): \delta 191.2,155.5,108.8,94.0,62.1,43.2,22.2$. HRMS. Calc'd for $\mathrm{C}_{8} \mathrm{H}_{11} \mathrm{NO}_{2}$ : 153.0790. Found 153.0789.

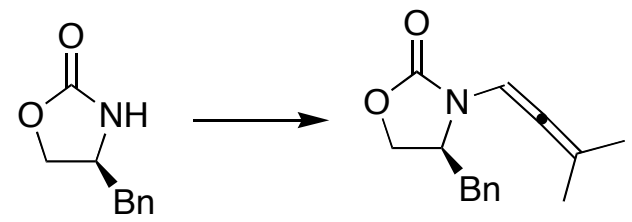

95\% yield. Isolated as an oil. $\mathrm{R}_{f}=0.42$ (ethyl acetate: petroleum ether 1:2). IR(neat): 2914, 1966, 1748, 1481, 1432, 1398, 1289, 1239, 1088, 998, 742, 702, $679 \mathrm{~cm}^{-1} .{ }^{1} \mathrm{H}$ NMR (400 MHz, $\left.\mathrm{CDCl}_{3}\right): \delta$ 7.24-7.35 (m, 3H), 7.10-7.16 (m, 2H), $6.66(\mathrm{sp}, J=2.59 \mathrm{~Hz}$, $1 \mathrm{H}), 4.21(\mathrm{dd}, J=8.69,8.69 \mathrm{~Hz}), 4.11(\mathrm{dd}, J=8.85,3.81 \mathrm{~Hz}), 4.00-4.08$ (m, 1H), 3.16 (dd, $J=13.57,2.90 \mathrm{~Hz}, 1 \mathrm{H}), 2.71(\mathrm{dd}, J=13.57,9.00 \mathrm{~Hz}, 1 \mathrm{H}), 1.90(\mathrm{~d}, 2.59 \mathrm{~Hz}, 3 \mathrm{H}), 1.88$ (d, 2.59Hz, 3H). ${ }^{13} \mathrm{C}$ NMR (101 MHz, $\left.\mathrm{CDCl}_{3}\right): \delta 191.7,155.1,135.5,129.1,128.8$, $127.1,108.5,93.0,66.3,55.6,36.5,22.0,21.8 .[\alpha]_{\mathrm{D}}^{24.2}=+11.89 \pm 0.03^{\circ}\left(\mathrm{c}=2.95, \mathrm{CH}_{2} \mathrm{Cl}_{2}\right)$. HRMS. Calc'd for $\mathrm{C}_{15} \mathrm{H}_{17} \mathrm{NO}_{2}$ : 243.1259. Found 243.1256.<smiles>CC(C)=C(C)C=CN1C(=O)O[C@@H](c2ccccc2)[C@H]1C</smiles> 
$99 \%$ yield. Isolated as an off-white solid ( $\mathrm{mp} 73-75^{\circ} \mathrm{C}$ ). $\mathrm{R}_{f}=0.37$ (ether: petroleum ether 1:2). IR(neat): 2982, 2913, 1966, 1756, 1479, 1428, 1376, 1354, 1230, 1108, 760, 701, $676 \mathrm{~cm}^{-1} .{ }^{1} \mathrm{H} \mathrm{NMR}\left(400 \mathrm{MHz}, \mathrm{CDCl}_{3}\right): \delta 7.25-7.40(\mathrm{~m}, 5 \mathrm{H}), 6.59(\mathrm{sp}, J=2.59 \mathrm{~Hz}, 1 \mathrm{H})$, $5.63(\mathrm{~d}, J=7.93 \mathrm{~Hz}, 1 \mathrm{H}), 4.11(\mathrm{dq}, J=6.56,1.37 \mathrm{~Hz}, 1 \mathrm{H}), 1.77(\mathrm{~d}, J=2.59 \mathrm{~Hz}, 3 \mathrm{H})$, $1.75(\mathrm{~d}, J=2.59,3 \mathrm{H}), 0.75(\mathrm{~d}, J=6.56 \mathrm{~Hz}, 3 \mathrm{H}) .{ }^{13} \mathrm{C} \mathrm{NMR}\left(101 \mathrm{MHz}, \mathrm{CDCl}_{3}\right): \delta 191.6$, $154.5,134.4,128.35,128.30,125.8,108.2,92.7,78.8,54.7,21.9,21.4,13.5 .[\alpha]_{\mathrm{D}}^{23.8}=-$ $35.39 \pm 0.12^{\circ}\left(\mathrm{c}=2.15, \mathrm{CH}_{2} \mathrm{Cl}_{2}\right)$. HRMS. Calc'd for $\mathrm{C}_{15} \mathrm{H}_{17} \mathrm{NO}_{2}: 243.1259$. Found 243.1254 .

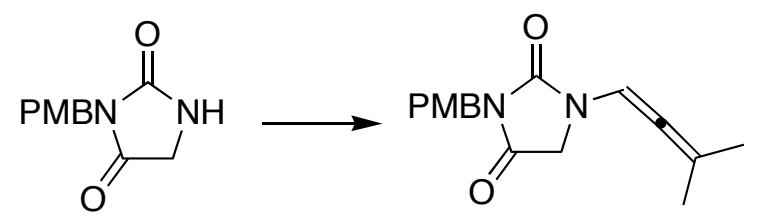

$99 \%$ yield. Isolated as an oil. $\mathrm{R}_{f}=0.43$ (ether: petroleum ether 1:1). $\operatorname{IR}$ (neat): 2937, 2838, 1966, 1772, 1715, 1514, 1479, 1449, 1352, 1298, 1248, 1225, 1177, 1141, 1109, 1033, $767 \mathrm{~cm}^{-1} .{ }^{1} \mathrm{H}$ NMR $\left(400 \mathrm{MHz}, \mathrm{CDCl}_{3}\right): \delta 7.37(\mathrm{~d}, J=8.69,2 \mathrm{H}), 6.83(\mathrm{~d}, J=8.69,2 \mathrm{H})$, 6.77 (sp, $J=2.59,1 \mathrm{H}), 4.60$ (s, 2H), 3.80 (s, 2H), 3.77 (s, 3H), 1.78 (d, $J=2.44 \mathrm{~Hz}, 6 \mathrm{H})$. ${ }^{13} \mathrm{C}$ NMR (101 MHz, $\left.\mathrm{CDCl}_{3}\right): \delta$ 190.9. 168.8, 159.3, 153.9, 130.3, 128.1, 113.9, 109.9, 92.4, 55.2, 48.5, 41.9, 22.1. HRMS. Calc'd for $\mathrm{C}_{16} \mathrm{H}_{18} \mathrm{~N}_{2} \mathrm{O}_{3}$ : 286.1317. Found 286.1310.

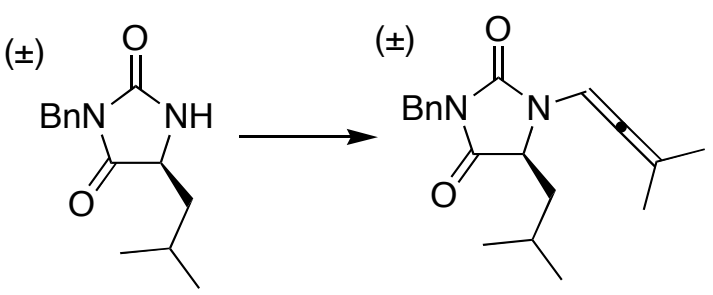

$100 \%$ yield. Isolated as an oil. $\mathrm{R}_{f}=0.33$ (ether: petroleum ether 1:4). $\operatorname{IR}$ (neat): 2958 , 1969, 1772, 1715, 1439, 1414, 1362, 1206, 1145, 749, $700 \mathrm{~cm}^{-1}$. ${ }^{1} \mathrm{H}$ NMR $(400 \mathrm{MHz}$, $\left.\mathrm{CDCl}_{3}\right): \delta$ 7.38-7.43 (m, 2H), 7.26-7.34 (m, 3H), $6.76(\mathrm{sp}, J=2.59 \mathrm{~Hz}, 1 \mathrm{H}), 4.66(\mathrm{~s}, 2 \mathrm{H})$, $3.96(\mathrm{dd}, J=8.24,3.04 \mathrm{~Hz}), 1.64-1.90$ (m, $10 \mathrm{H}), 0.89$ (d, $J=6.56 \mathrm{~Hz}, 3 \mathrm{H}), 0.78$ (d, $J=$ $6.41 \mathrm{~Hz}, 3 \mathrm{H}) .{ }^{13} \mathrm{C}$ NMR $\left(101 \mathrm{MHz}, \mathrm{CDCl}_{3}\right): \delta 191.52,172.6,153.8,135.7,128.8,128.5$, 127.9, 108.7, 91.5, 57.9, 42.4, 37.2, 23.7, 23.6, 21.94, 21.86, 21.69. HRMS. Calc'd for $\mathrm{C}_{19} \mathrm{H}_{24} \mathrm{~N}_{2} \mathrm{O}_{2}: 312.1838$. Found 312.1828. 


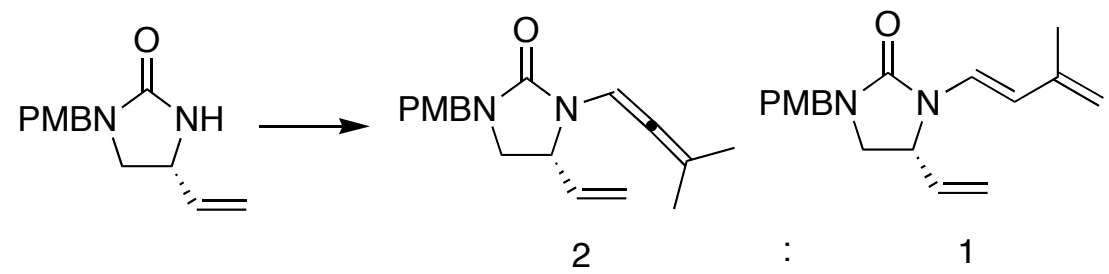

$79 \%$ yield. Isolated as an oil. Data for inseparable mixture of compounds: $\mathrm{R}_{f}=0.30$ (ether: petroleum ether 1:1). IR(neat): 2910, 1966, 1706, 1644, 1612, 1514, 1442, 1247, 1176, 1035, 945, 820, $747 \mathrm{~cm}^{-1} .{ }^{1} \mathrm{H}$ NMR (400 MHz, $\left.\mathrm{CDCl}_{3}\right): \delta$ 7.15-7.20 (m, 2H), 6.97 (d, $J=14.79 \mathrm{~Hz}, 0.33 \mathrm{H}), 6.82-6.88(\mathrm{~m}, 2 \mathrm{H}), 6.72(\mathrm{sp}, J=2.59 \mathrm{~Hz}, 0.66 \mathrm{H}), 5.56-5.80$ (m, $1.33 \mathrm{H}), 5.07-5.30(\mathrm{~m}, 2 \mathrm{H}), 4.73(\mathrm{~m}, 0.66 \mathrm{H}), 4.28-4.38(\mathrm{~m}, 2.33 \mathrm{H}), 4.04(\mathrm{~m}, 0.66 \mathrm{H}), 3.78$ (s, 3H), 3.48 (t, $J=9.30 \mathrm{~Hz}, 0.33 \mathrm{H}), 3.40$ (t, $J=9.15 \mathrm{~Hz}, 0.66 \mathrm{H}), 2.96$ (dd, $J=9.15,4.42$ $\mathrm{Hz}, 0.33 \mathrm{H}), 2.90$ (dd, $J=9.15,3.66 \mathrm{~Hz}, 0.66 \mathrm{H}), 1.87$ (s, 1H), 1.70 (d, $J=2.59 \mathrm{~Hz}, 2 \mathrm{H}$ ), $1.64(\mathrm{~d}, J=2.59 \mathrm{~Hz}, 2 \mathrm{H}) .{ }^{13} \mathrm{C} \mathrm{NMR}\left(101 \mathrm{MHz}, \mathrm{CDCl}_{3}\right): \delta 191.7,159.0,158.9,157.1$, 156.5, 141.0, 136.5, 136.2, 129.4, 129.3, 128.7, 128.3, 124.8, 117.6, 117.0, 114.0, 113.9, $111.9,111.1,106.8,93.9,55.23,55.17,54.3,48.3,48.1,47.2,47.1,22.4,22.3,18.8$. $[\alpha]_{\mathrm{D}}^{22.5}=-1.23 \pm 0.09^{\circ}\left(\mathrm{c}=1.76, \mathrm{CH}_{2} \mathrm{Cl}_{2}\right)$. HRMS. Calc'd for $\mathrm{C}_{18} \mathrm{H}_{22} \mathrm{~N}_{2} \mathrm{O}_{2}: 298.1681$. Found 298.1679.

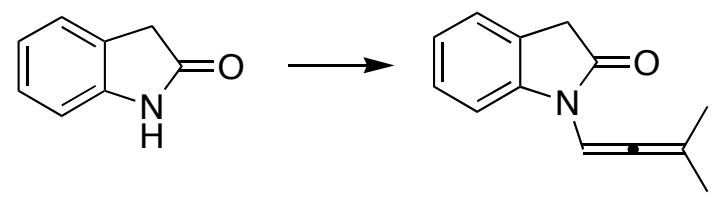

$86 \%$ yield. Isolated as a yellow solid $\left(\mathrm{mp} 90-92^{\circ} \mathrm{C}\right) . \mathrm{R}_{f}=0.30$ (ether: petroleum ether 1:2). IR(neat): 2909, 1967, 1716, 1613, 1491, 1467, 1375, 1306, 1253, 1175, 1084, 748 $\mathrm{cm}^{-1} .{ }^{1} \mathrm{H}$ NMR (400 MHz, $\left.\mathrm{CDCl}_{3}\right): \delta$ 7.21-7.30 (m, 3H), 7.03-7.08 (m, 1H), 6.99 (sp, $J=$ $2.59 \mathrm{~Hz}, 1 \mathrm{H}), 3.53(\mathrm{~s}, 2 \mathrm{H}), 1.90(\mathrm{~d}, J=2.75 \mathrm{~Hz}, 6 \mathrm{H}) .{ }^{13} \mathrm{C} \mathrm{NMR}\left(101 \mathrm{MHz}, \mathrm{CDCl}_{3}\right)$ : $\delta$ 193.7, 173.4, 143.1, 127.8, 124.5, 124.0, 122.7, 110.3, 107.2, 89.7, 35.0, 21.9. HRMS. Calc'd for $\mathrm{C}_{13} \mathrm{H}_{13} \mathrm{NO}$ : 199.0997. Found 199.1002. 


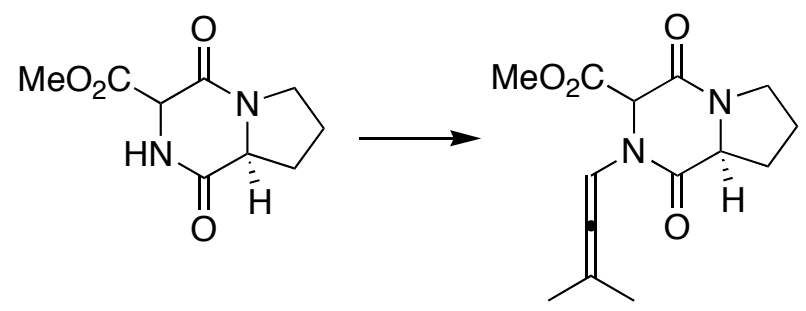

$63 \%$ yield. Isolated as oil. $\mathrm{R}_{f}=0.40$ (5\% triethylamine in ethyl acetate). IR(neat): 2954 , 1968, 1748, 1682, 1428, 1387, 1279, $1190 \mathrm{~cm}^{-1} .{ }^{1} \mathrm{H}$ NMR (400 MHz, $\left.\mathrm{CDCl}_{3}\right): \delta 7.08$ (sp, $J=2.44 \mathrm{~Hz}, 1 \mathrm{H}), 4.83(\mathrm{~s}, 1 \mathrm{H}), 4.28(\mathrm{~m}, 1 \mathrm{H}), 3.79(\mathrm{~s}, 3 \mathrm{H}), 3.52-3.62(\mathrm{~m}, 2 \mathrm{H}), 2.42(\mathrm{~m}$, $1 \mathrm{H}), 1.84-2.10(\mathrm{~m}, 3 \mathrm{H}), 1.77(\mathrm{~d}, J=2.59 \mathrm{~Hz}, 3 \mathrm{H}), 1.70(\mathrm{~d}, J=2.44,3 \mathrm{H}) .{ }^{13} \mathrm{C}$ NMR $(101$ $\mathrm{MHz}_{\mathrm{CDCl}}$ ): $\delta 191.2,166.4,165.5,158.6,111.3,94.7,63.5,59.0,53.3,46.0,28.9$,

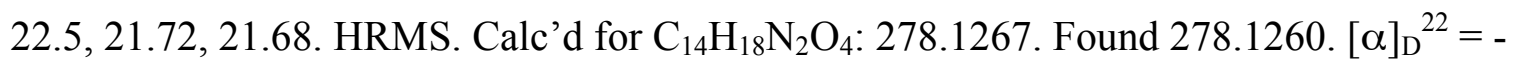
$1.75 \pm 0.53^{\circ}\left(\mathrm{c}=3.90, \mathrm{CH}_{2} \mathrm{Cl}_{2}\right)$.

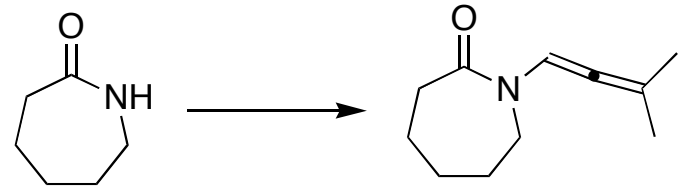

$46 \%$ yield. Isolated as an oil. $\mathrm{R}_{f}=0.32$ (ether: petroleum ether 3:1). IR(neat): 2930, 1964, 1659, 1471, 1441, 1390, 1275, 1190, 1148, 1082, 973, $896 \mathrm{~cm}^{-1}$. ${ }^{1} \mathrm{H}$ NMR (400 MHz, $\left.\mathrm{CDCl}_{3}\right): \delta 7.14(\mathrm{sp}, J=2.44 \mathrm{~Hz}, 1 \mathrm{H}), 3.41(\mathrm{~m}, 2 \mathrm{H}), 2.55(\mathrm{~m}, 2 \mathrm{H}), 1.76(\mathrm{~d}, J=2.59 \mathrm{~Hz}$, $6 \mathrm{H}), 1.54-1.72(\mathrm{~m}, 6 \mathrm{H}) .{ }^{13} \mathrm{C} \mathrm{NMR}\left(101 \mathrm{MHz}, \mathrm{CDCl}_{3}\right): \delta 191.4,173.9,108.0,97.0,45.8$, 37.3, 29.7, 27.4, 23.4, 21.9. HRMS. Calc'd for $\mathrm{C}_{11} \mathrm{H}_{17} \mathrm{NO}$ : 179.1310. Found 179.1311.

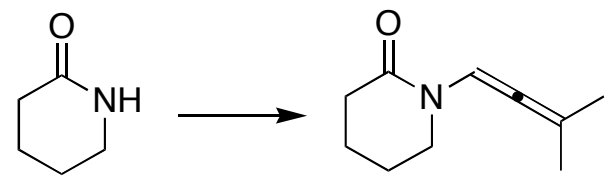

28\% yield. Isolated as an oil. $\mathrm{R}_{f}=0.25$ (ether: petroleum ether 3:1). IR(neat): 2940, 1967, 1654, 1484, 1433, 1346, 1306, 1190, 1176, 1092, 982, $821 \mathrm{~cm}^{-1}$. ${ }^{1} \mathrm{H}$ NMR (200 MHz, $\left.\mathrm{CDCl}_{3}\right): \delta 7.30(\mathrm{sp}, J=2.56 \mathrm{~Hz}, 1 \mathrm{H}), 3.21(\mathrm{~m}, 2 \mathrm{H}), 2.41(\mathrm{~m}, 2 \mathrm{H}), 1.65-1.85(\mathrm{~m}, 10 \mathrm{H})$.

${ }^{13} \mathrm{C} \mathrm{NMR}\left(50 \mathrm{MHz}, \mathrm{CDCl}_{3}\right): \delta$ 192.2, 168.2, 107.5, 96.5, 45.7, 32.7, 22.6, 22.0, 20.9. HRMS. Calc'd for $\mathrm{C}_{10} \mathrm{H}_{15} \mathrm{NO}$ : 165.1154. Found 165.1158. 


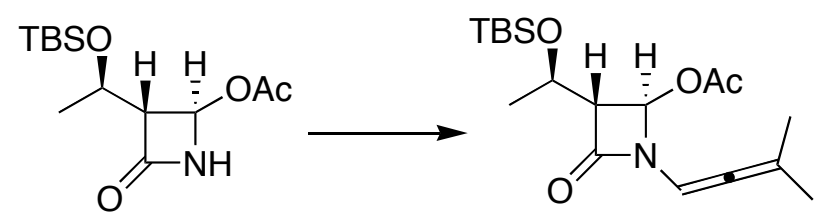

$36 \%$ yield. Isolated as an oil. $\mathrm{R}_{f}=0.28$ (ether: petroleum ether, 1:3). IR(neat): 2931, 2858, 1968, 1777, 1472, 1429, 1376, 1332, 1227, 1075, 1033, 838, $778 \mathrm{~cm}^{-1}$. ${ }^{1} \mathrm{H}$ NMR (400 MHz, $\left.\mathrm{CDCl}_{3}\right): \delta 6.25-6.28(\mathrm{~m}, 2 \mathrm{H}), 4.20(\mathrm{~m}, 1 \mathrm{H}), 3.07(\mathrm{dd}, J=2.75,1.07 \mathrm{~Hz}, 1 \mathrm{H})$, $2.07(\mathrm{~s}, 3 \mathrm{H}), 1.75(\mathrm{~d}, J=2.59 \mathrm{~Hz}, 3 \mathrm{H}), 1.73(\mathrm{~d}, J=2.59 \mathrm{~Hz}, 3 \mathrm{H}), 1.24(\mathrm{~d}, J=6.41 \mathrm{~Hz}$, 3H), 0.84 (s, 9H), 0.05 (s, 3H), 0.04 (s, 3H). $\left.{ }^{13} \mathrm{C} \mathrm{NMR} \mathrm{(101} \mathrm{MHz,} \mathrm{CDCl}_{3}\right): \delta$ 192.1, 169.6, 164.2, 108.5, 89.1, 76.5, 65.3, 63.9, 25.6, 22.1, 21.6, 21.2, 20.8, 17.8, -4.2, -5.4. $[\alpha]_{\mathrm{D}}^{22.3}=+28.99 \pm 0.07^{\circ}\left(\mathrm{c}=2.29, \mathrm{CH}_{2} \mathrm{Cl}_{2}\right)$. HRMS. Calc'd for $\mathrm{C}_{18} \mathrm{H}_{31} \mathrm{NO}_{4} \mathrm{Si}:$ 353.2022. Found 353.2029.

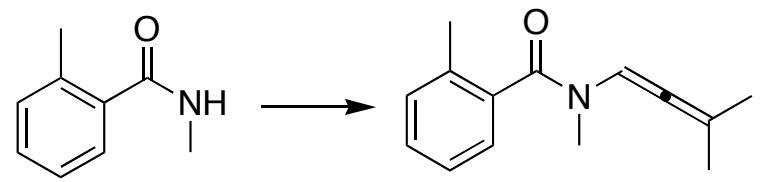

$20 \%$ yield. Isolated as an oil. Data for 2:1 mixture of amide rotomers: $\mathrm{R}_{f}=0.32$ (ether: petroleum ether, 1:2). IR(neat): 2931, 1963, 1652, 1481, 1346, 1285, 1061, $739 \mathrm{~cm}^{-1} .{ }^{1} \mathrm{H}$ NMR (200 MHz, $\left.\mathrm{CDCl}_{3}\right): \delta 7.46(\mathrm{sp}, J=2.56 \mathrm{~Hz}, 0.33 \mathrm{H}), 7.17-7.33$ (m, 4H), 6.20 (sp, $J$ $=2.56 \mathrm{~Hz}, 0.66 \mathrm{H}), 3.12(\mathrm{~s}, 2 \mathrm{H}), 2.78(\mathrm{~s}, 1 \mathrm{H}), 2.31(\mathrm{~s}, 1 \mathrm{H}), 2.29(\mathrm{~s}, 2 \mathrm{H}), 1.82(\mathrm{~d}, J=2.56$ $\mathrm{Hz}, 2 \mathrm{H}), 1.76$ (d, $J=2.56 \mathrm{~Hz}, 4 \mathrm{H}) .{ }^{13} \mathrm{C} \mathrm{NMR}\left(101 \mathrm{MHz}, \mathrm{CDCl}_{3}\right): \delta 190.8,135.7,134.5$, $130.38,130.35,129.1,129.0,126.5,125.97,125.95,125.88,107.8,99.7,96.7,34.4,30.4$, 25.1, 22.1, 19.03, 18.97. HRMS. Calc'd for $\mathrm{C}_{14} \mathrm{H}_{17} \mathrm{NO}: 215.1310$. Found 215.1310.<smiles>C=C(C)/C=C/NC(=O)c1ccccc1</smiles>

$47 \%$ yield. Isolated as a yellow solid ( $\mathrm{mp} 133-137^{\circ} \mathrm{C}$ ). $\mathrm{R}_{f}=0.52$ (ethyl acetate: petroleum ether, 1:2). IR(neat): 3288, 2918, 1636, 1525, 1490, 1314, 1192, 954, $705 \mathrm{~cm}^{-1}$. ${ }^{1} \mathrm{H}$ NMR $\left(500 \mathrm{MHz}, \mathrm{CDCl}_{3}\right): \delta 8.04(\mathrm{~d}, J=9.77 \mathrm{~Hz}, 1 \mathrm{H}), 7.82(\mathrm{~m}, 2 \mathrm{H}), 7.53(\mathrm{~m}, 1 \mathrm{H}), 7.44(\mathrm{~m}$, 2H), $7.20(\mathrm{dd}, J=14.40,10.87 \mathrm{~Hz}, 1 \mathrm{H}), 6.08$ (d, $J=14.40 \mathrm{~Hz}, 1 \mathrm{H}), 4.87$ (s, 2H), 1.90 (s, 
3H). ${ }^{13} \mathrm{C}$ NMR (126 MHz, $\left.\mathrm{CDCl}_{3}\right): \delta 164.5,140.4,133.5,132.0,128.7,127.1,123.1$, 117.0, 114.5, 18.7. HRMS. Calc'd for $\mathrm{C}_{12} \mathrm{H}_{13} \mathrm{NO}$ : 187.0997. Found 187.0990.

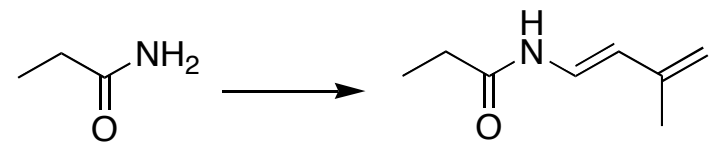

$24 \%$ yield. Isolated as an oil. $\mathrm{R}_{f}=0.45$ (ethyl acetate: petroleum ether, $\left.1: 2\right)$. IR(neat): $3312,2979,2929,1648,1490,1375,1272,1200,1109 \mathrm{~cm}^{-1}$. ${ }^{1} \mathrm{H}$ NMR $(400 \mathrm{MHz}$, $\left.\mathrm{CDCl}_{3}\right): \delta 7.64(\mathrm{~s}, 1 \mathrm{H}), 6.81(\mathrm{dd}, J=11.29,9.76 \mathrm{~Hz}, 1 \mathrm{H}), 5.10(\mathrm{~d}, J=9.76 \mathrm{~Hz}, 1 \mathrm{H}), 5.07$ (s, 1H), 4.93 (s, 1H), 2.28 (q, $J=7.63 \mathrm{~Hz}, 2 \mathrm{H}), 1.88$ (s, 3H), 1.18 (t, $J=7.63 \mathrm{~Hz}, 3 \mathrm{H})$.

${ }^{13} \mathrm{C}$ NMR (101 MHz, $\left.\mathrm{CDCl}_{3}\right)$ : $\delta$ 170.9, 140.7, 121.3, 114.0, 111.2, 29.8, 23.7, 9.4. HRMS. Calc'd for $\mathrm{C}_{8} \mathrm{H}_{13} \mathrm{NO}$ : 139.0997. Found 139.0997.

\section{Variations of general procedure:}

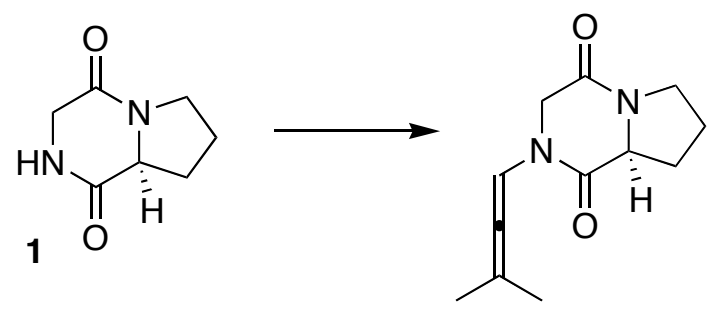

An oven-dried $10 \mathrm{~mL}$ roundbottom flask was charged with diketopiperazine 1 (198 mg, $1.29 \mathrm{mmol}), \mathrm{CuI}(12 \mathrm{mg}, 64 \mu \mathrm{mol})$, and $\mathrm{Cs}_{2} \mathrm{CO}_{3}(628 \mathrm{mg}, 1.93 \mathrm{mmol})$. After flushing with nitrogen 3 times, $2 \mathrm{~mL}$ of DMF was added, followed by trans $-N, N$ 'dimethylcyclohexyldiamine ( $20 \mu \mathrm{L}, 18 \mathrm{mg}, 129 \mu \mathrm{mol})$, then 1-iodo-3-methylbuta-1,2diene ( $230 \mu \mathrm{L}, 374 \mathrm{mg}, 1.93 \mathrm{mmol})$. The flask was covered with aluminum foil and heated to $75^{\circ} \mathrm{C}$ for $16 \mathrm{~h}$. The reaction was cooled, added to $10 \mathrm{~mL}$ of water, extracted with ethyl acetate $(3 \times 5 \mathrm{~mL})$, washed with water, brine, then dried over magnesium sulfate and evaporated. The residue was purified by column chromatography on silica gel (95\% ethyl acetate, $5 \%$ triethylamine) to afford the allenamide (166 mg, 58\%) as an oil. $\mathrm{R}_{f}=0.31$ (95\% ethyl acetate, 5\% triethylamine). IR (neat): 2982, 1965, 1673, 1447, 1390, 1266, 1189. ${ }^{1} \mathrm{H} \mathrm{NMR}\left(\mathrm{CDCl}_{3}, 400 \mathrm{MHz}\right) \delta 7.00$ (sp, 1H, $\left.J=2.8 \mathrm{~Hz}\right), 4.07$ (m, 1H), 3.94 (d, $1 \mathrm{H}, J=17.2 \mathrm{~Hz}), 3.84(\mathrm{~d}, 1 \mathrm{H}, J=17.2 \mathrm{~Hz}), 3.40-3.58(\mathrm{~m}, 2 \mathrm{H}), 2.28-2.36(\mathrm{~m}, 1 \mathrm{H})$, 1.92-2.00 (m, 2H), 1.80-1.88 (m, 1H), $1.70(\mathrm{~d}, 6 \mathrm{H}, J=2.8 \mathrm{~Hz}) .{ }^{13} \mathrm{C} \mathrm{NMR}\left(\mathrm{CDCl}_{3}, 101\right.$ 
MHz) $\delta 191.6,164.7,162.6,109.8,94.6,58.9,49.0,45.0,28.7,22.3,21.7,21.6 . ~ H R M S$.

Calc'd for $\mathrm{C}_{12} \mathrm{H}_{16} \mathrm{~N}_{2} \mathrm{O}_{2}: 220.1212$. Found 220.1204. $[\alpha]_{\mathrm{D}}^{25}=-1.38$, (c 11.63, $\left.\mathrm{CH}_{2} \mathrm{Cl}_{2}\right)$.

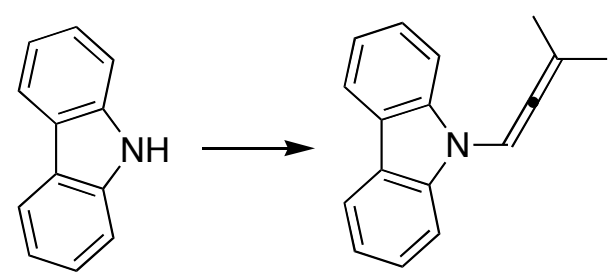

An oven-dried $10 \mathrm{~mL}$ roundbottom flask was charged with carbazole ( $84 \mathrm{mg}, 0.5 \mathrm{mmol}$ ), CuTC (6.7 mg, $35 \mu \mathrm{mol}), \mathrm{BaO}(153 \mathrm{mg}, 1 \mathrm{mmol})$ and $\mathrm{K}_{3} \mathrm{PO}_{4}(218 \mathrm{mg}, 1 \mathrm{mmol})$. After flushing with nitrogen 3 times, $2.5 \mathrm{~mL}$ of toluene was added, followed by trans $-N, N$ 'dimethylcyc9ohexyldiamine ( $12 \mu \mathrm{L}, 11 \mathrm{mg}, 75 \mu \mathrm{mol})$, then 1-iodo-3-methylbuta-1,2diene (101 $\mu \mathrm{L}, 165 \mathrm{mg}, 0.85 \mathrm{mmol})$. The flask was covered with aluminum foil and heated to $85^{\circ} \mathrm{C}$ for $16 \mathrm{~h}$. The reaction was cooled, diluted with $5 \mathrm{~mL}$ of ethyl acetate, filtered through a plug of celite and concentrated under vacuum. The residue was purified by column chromatography on silica gel (petroleum ether: ether: triethylamine, 95:2:3) to afford the allene (110 mg, 94\%) as an oil. $\mathrm{R}_{f}=0.47$ (petroleum ether: ether: triethylamine, 95:2:3). IR(neat): 3052, 2907, 1964, 1625, 1599, 1494, 1454, 1311, 1255, $1218,1153,1072,1004,922,747,720 \mathrm{~cm}^{-1} .{ }^{1} \mathrm{H}$ NMR $\left(400 \mathrm{MHz}, \mathrm{CDCl}_{3}\right): \delta 8.13(\mathrm{~m}$, 2H), 7.69 (m, 2H), $7.54(\mathrm{dt}, J=7.15,1.22 \mathrm{~Hz}, 2 \mathrm{H}), 7.33$ (dt, $J=7.17,0.92 \mathrm{~Hz}, 2 \mathrm{H}), 7.22$ $(\mathrm{sp}, J=2.59,1 \mathrm{H}), 2.05$ (d, $J=2.75,6 \mathrm{H}) .{ }^{13} \mathrm{C} \mathrm{NMR}\left(101 \mathrm{MHz}, \mathrm{CDCl}_{3}\right): \delta$ 195.1, 139.4, 125.9, 123.7, 120.1, 119.9, 110.0, 106.9, 92.3, 22.3. HRMS. Calc'd for $\mathrm{C}_{17} \mathrm{H}_{15} \mathrm{~N}$ :

233.1204. Found 233.1204.

\section{General procedure for coupling with 1-iodo-1,2-hexadiene:}

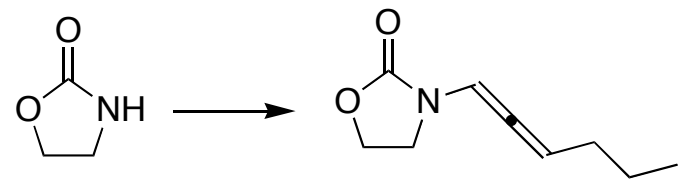

An oven-dried $10 \mathrm{~mL}$ roundbottom flask was charged with 2-oxazolidinone (35 mg, 0.40 $\mathrm{mmol}$ ), $\mathrm{CuTC}$ (5.3 mg, $28 \mu \mathrm{mol})$, and $\mathrm{K}_{3} \mathrm{PO}_{4}(170 \mathrm{mg}, 0.80 \mathrm{mmol})$. After flushing with nitrogen 3 times, $2 \mathrm{~mL}$ of toluene was added, followed by trans $-N, N^{\prime}$ - 
dimethylcyclohexyldiamine $(9.5 \mu \mathrm{L}, 8.5 \mathrm{mg}, 60 \mu \mathrm{mol})$, then 1-iodo-1,2-hexadiene (100 $\mu \mathrm{L}, 141 \mathrm{mg}, 0.68 \mathrm{mmol})$. The flask was covered with aluminum foil and heated to $85^{\circ} \mathrm{C}$ for $5 \mathrm{~h}$. The reaction was cooled and added to $10 \mathrm{~mL}$ of water, then extracted with ethyl acetate $(3 \times 5 \mathrm{~mL})$, washed with brine, dried over magnesium sulfate, and concentrated under vacuum. The residue was purified by column chromatography on silica gel (eluted with ether: petroleum ether, $2: 1+5 \%$ triethylamine) to afford the allenamide (67 mg, $100 \%$ ) as an oil. $\mathrm{R}_{f}=0.37$ (ether: petroleum ether, 2:1). $\mathrm{IR}$ (neat): 2959, 1965, 1754, 1483, 1455, 1395, 1285, 1233, 1066, 1030, $753 \mathrm{~cm}^{-1} .{ }^{1} \mathrm{H}$ NMR (400 MHz, $\left.\mathrm{CDCl}_{3}\right): \delta 6.73$ (dt, $J$ $=5.95,2.75 \mathrm{~Hz}, 1 \mathrm{H}), 5.78(\mathrm{q}, J=6.56 \mathrm{~Hz}, 1 \mathrm{H}), 4.37(\mathrm{t}, J=7.47 \mathrm{~Hz}, 2 \mathrm{H}), 3.55(\mathrm{~m}, 2 \mathrm{H})$, $2.02(\mathrm{~m}, 2 \mathrm{H}), 1.41(\mathrm{~m}, 2 \mathrm{H}), 0.89(\mathrm{t}, J=7.32 \mathrm{~Hz}, 3 \mathrm{H}) .{ }^{13} \mathrm{C} \mathrm{NMR}\left(101 \mathrm{MHz}, \mathrm{CDCl}_{3}\right)$ : $\delta$ 194.6, 155.3, 104.0, 96.5, 62.1, 43.1, 32.3, 21.7, 13.5. HRMS. Calc'd for $\mathrm{C}_{9} \mathrm{H}_{13} \mathrm{NO}_{2}$ : 167.0946. Found 167.0943.

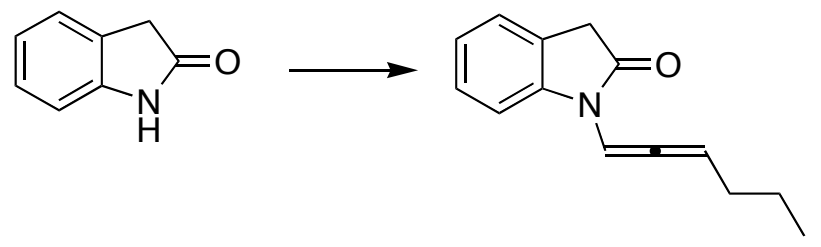

$100 \%$ yield. Isolated as an oil. $\mathrm{R}_{f}=0.32$ (ether: petroleum ether, 1:3). $\operatorname{IR}$ (neat): 2959 , 1963, 1720, 1612, 1489, 1467, 1377, 1313, 1239, 1198, 1178, $748 \mathrm{~cm}^{-1} .{ }^{1} \mathrm{H}$ NMR (400 $\left.\mathrm{MHz}, \mathrm{CDCl}_{3}\right): \delta 7.45(\mathrm{~m}, 1 \mathrm{H}), 7.25(\mathrm{~m}, 2 \mathrm{H}), 7.16(\mathrm{dt}, J=6.25,3.05 \mathrm{~Hz}, 1 \mathrm{H}), 7.07(\mathrm{~m}$, 1H), 5.94 (q, $J=6.41 \mathrm{~Hz}, 1 \mathrm{H}), 3.54(\mathrm{~s}, 2 \mathrm{H}), 2.20(\mathrm{~m}, 2 \mathrm{H}), 1.55(\mathrm{~m}, 2 \mathrm{H}), 0.98$ (t, $J=7.47$ $\mathrm{Hz}) .{ }^{13} \mathrm{C} \mathrm{NMR}\left(101 \mathrm{MHz}, \mathrm{CDCl}_{3}\right): \delta 196.8,173.2,143.0,127.7,124.4,124.1,122.8$, 110.8, 103.0, 92.0, 34.9, 32.2, 21.7, 13.7. HRMS. Calc'd for $\mathrm{C}_{14} \mathrm{H}_{15} \mathrm{NO}$ : 213.1154. Found 213.1152 .

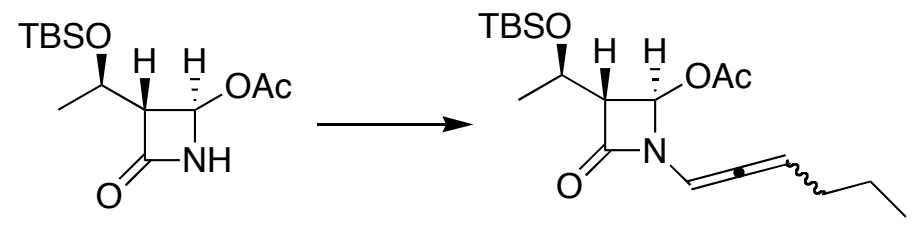

$47 \%$ yield. Isolated as an oil. Data for 1:1 mixture of diastereomers: $\mathrm{R}_{f}=0.33$ (ether: petroleum ether, 1:3). IR(neat): 2959, 2858, 1965, 1779, 1455, 1377, 1212, 1178, 1073, 1034, 838, $778 \mathrm{~cm}^{-1} .{ }^{1} \mathrm{H}$ NMR (400 MHz, $\left.\mathrm{CDCl}_{3}\right): \delta$ 6.40-6.46 (m, $\left.1 \mathrm{H}\right), 6.28(\mathrm{~m}, 1 \mathrm{H})$, 
5.70-5.78 (m, 1H), $4.21(\mathrm{~m}, 1 \mathrm{H}), 3.08(\mathrm{~m}, 1 \mathrm{H}), 1.98-2.10(\mathrm{~m}, 5 \mathrm{H}), 1.42(\mathrm{~m}, 2 \mathrm{H}), 1.24(\mathrm{~d}$, $J=6.41 \mathrm{~Hz}, 3 \mathrm{H}), 0.92(\mathrm{t}, J=7.32 \mathrm{~Hz}, 3 \mathrm{H}), 0.83(\mathrm{~m}, 9 \mathrm{H}), 0.05(\mathrm{~m}, 6 \mathrm{H}) .{ }^{13} \mathrm{C}$ NMR $(101$ $\left.\mathrm{MHz}, \mathrm{CDCl}_{3}\right): \delta 195.1,169.6,169.5,164.2,103.5,103.3,91.8,91.7,77.3,77.1,77.0$, 76.8, 76.7, 65.72, 65.69, 64.0, 63.9, 31.9, 31.8, 25.7, 25.6, 22.2, 22.1, 21.6, 21.1, 20.8, $17.8,13.64,13.59,-4.27,-4.29,-5.33,-5.37 .[\alpha]_{\mathrm{D}}^{25.0}=+8.10 \pm 0.07^{\circ}\left(\mathrm{c}=3.39, \mathrm{CH}_{2} \mathrm{Cl}_{2}\right)$. HRMS. Calc'd for $\mathrm{C}_{19} \mathrm{H}_{33} \mathrm{NO}_{4} \mathrm{Si}: 367.2179$. Found 367.2168.

${ }^{1}$ Greaves, P. M.; Kalli, M.; Landor, P. D.; Landor, S. R. J. Chem. Soc. (C) 1971, 667.

${ }^{2}$ Landor, S. R.; Patel, A. N.; Whiter, P. F.; Greaves, P. M. J. Chem. Soc. (C) 1966, 1223. 